\section{Detection of fetal cells in transcervical samples using X22 marker}

EDITOR-The presence of trophoblastic cells in the endocervical canal of pregnant women between 5-7 and 13-15 weeks of gestation has been repeatedly confirmed. ${ }^{12}$ Using fluorescence in situ hybridisation (FISH) or the polymerase chain reaction (PCR) assay, chromosome Y derived sequences have been detected in trancervical cells (TCCs) retrieved from mothers with male fetuses.

Although small tandem repeats (STRs) and quantitative fluorescent PCR (QF-PCR) have also been used to monitor the presence in TCC samples of fetal DNA sequences inherited from the father and absent in the mothers, ${ }^{13}$ the direct and unequivocal demonstration of trophoblastic cells derived from female fetuses has been hampered by the unavailability of highly polymorphic markers specific for the $\mathrm{X}$ and $\mathrm{Y}$ chromosomes.

In this pilot study, we have assessed the diagnostic value of using a new $\mathrm{X} / \mathrm{Y}$ chromosome marker, X22, ${ }^{4}$ for the detection of trophoblastic cellular elements released into the endocervical canal by female fetuses.

After receiving verbal consent, samples were retrieved by cervical mucus aspiration ${ }^{1}$ from four pregnant women, at about 10 weeks of gestation, before termination of pregnancy. Maternal peripheral blood and chorionic tissues were also collected. Aliquots of TCC samples were suspended in phosphate buffered saline (PBS) and analysed under an inverted microscope in order to isolate clumps of cells with the morphological characteristics of syncytial or cytotrophoblastic cellular elements.

DNA, extracted from chorionic tissue, individual clumps of TCC cells, and maternal blood samples, was then tested using single or multiplex QF-PCR assay and STRs specific for chromosomes 21, 18, and 13 besides the amelogenin (AMXY) and hypoxantine-guanil-phosporibosyl transferase (HPRT) markers for sexing. ${ }^{5}$ All samples were tested with the highly polymorphic X22 pentanucleotide (AAATA) repeat that maps in the PAR2 region of homology of the $\mathrm{X}$ and $\mathrm{Y}$ chromosomes. ${ }^{4}$ Previous investigations have documented that, when tested by QF-PCR, about $80 \%$ of samples from normal female subjects show two fluorescent PCR products corresponding to two allelic markers, thus allowing the diagnosis of heterozygosity. ${ }^{4}$

In this study, to enable fluorescence PCR analysis of the X22 marker in the TCC samples, the forward primer (taatgagagttggaaagaaa) was $5^{\prime}$ end labelled with 4, 7, 2', 7'tetrachloro-6-carboxyfluorescein while the reverse primer (cccattgttgctacttgaga) was unlabelled. PCR amplification was performed for 25 cycles at the following temperatures: $95^{\circ} \mathrm{C}$ for 45 seconds, $55^{\circ} \mathrm{C}$ for 45 seconds, and $72^{\circ} \mathrm{C}$ for one minute. The amplification products were sized and their relative fluorescent intensities calculated using an ABI 310 DNA scanner and Genescan software.

Table 1 show the results of testing maternal blood, chorion, and clumps of TCCs collected from two pregnant women. Both fetuses were females according to the AMXY tests performed on chorionic tissue. Mother HW was homozygous for the X22 marker (size 223); the chorionic tissue showed both maternal (223) and paternal (218) allelic X22 markers. Four TCC clumps (1, 2, 5, and 6) contained only maternal cells, while the paternal X22 marker 218 was present in the other three clumps $(3,4$, and 7 ), thus documenting the presence of trophoblastic cells from a female fetus. However, according to the QF-PCR multiplex assays performed using autosomal STR markers, only clump 7 was free of maternal contaminating cells.

Mother PG was homozygous for X22 (228 and 232), while the chorionic tissue documented the presence of a maternal (228) and paternal (204) marker. Two clumps (1 and 3 ) contained only maternal cells; clump 2 showed three fluorescent peaks (sizes 204, 224, and 228) which indicated the presence of both maternal and fetal cells. One clump (4) produced one maternal and one paternal X22 fluorescent PCR products (204 and 228) with a pattern similar to that detected in placental tissue. This result suggests that clump 4 contained only fetal cells, in agreement with the tests performed using the other chromosome markers

The other two mothers (cases 3 and 4) had male fetuses and in both cases $60 \%$ of the collected TCC clumps contained fetal cells. Fig $1 \mathrm{~A}$ shows the electrophoretogram of maternal blood, CVS, and one TCC clump of cells (case 3) tested with X22, AMXY, HPRT, and other STR markers. The X22 pattern in CVS and TCC clumps showed two peaks of maternal and paternal origin in addition to the chromosome Y marker. STRs for chromosomes 18 and 21 confirmed the presence of fetal cells in the clump. The maternal sample had two peaks for the HPRT while the CVS and clump contained only one of the maternally derived X chromosome markers. Fig 1B documents the diagnostic value of using only X22 and HPRT for the detection of fetal cells in TCC samples.

The present results confirm that trophoblastic cells from female fetuses are present in TCC samples and that clumps of fetal cells can be isolated by micromanipulation. Using the X22, AMXY, and HPRT markers and eventually other autosomal STRs for chromosomes 21, 18, and 13, the absence of maternal cell contaminants in some selected clumps can be readily established. The DNA extracted

Table 1 Maternal blood, CVS, and TCC samples tested by QF-PCR with X22 and other STR markers. All the mixed (fetal + maternal) clumps have skewed fluorescent peak ratios between the STRs alleles

\begin{tabular}{lllllll}
\hline Sample & AMXY & X22 & HPRT & D21S1411 & D13S634 & D18S386 \\
\hline H W (mat blood) & XX & 223 & 284 & 282 & $474-488$ & $352-376$ \\
CVS & XX & $218-223$ & $276-284$ & 282 & $474-482$ & $350-376$ \\
TCC clumps 1,2,5,6 & XX & 223 & 284 & 282 & $474-488$ & $352-376$ \\
TCC clumps 3,4 & XX & $218-223$ & $276-284$ & 282 & $474-482-488$ & $350-352-376$ \\
HTC clump 7 & XX & $218-223$ & $276-284$ & 282 & $474-482$ & $350-376$ \\
P G (mat blood) & XX & $228-233$ & 284 & $290-298$ & $478-482$ & 372 \\
CVS & XX & $203-228$ & $284-288$ & 290 & $466-478$ & $372-376$ \\
TCC clumps 1,3 & XX & $228-233$ & 284 & $290-298$ & $478-482$ & 372 \\
TCC clump 2 & XX & $203-228-223$ & $284-288$ & $290-298$ & $466-478$ & $372-376$ \\
TCC clump 4 & XX & $203-228$ & $284-288$ & 290 & $466-478$ & $372-376$ \\
\hline
\end{tabular}


A

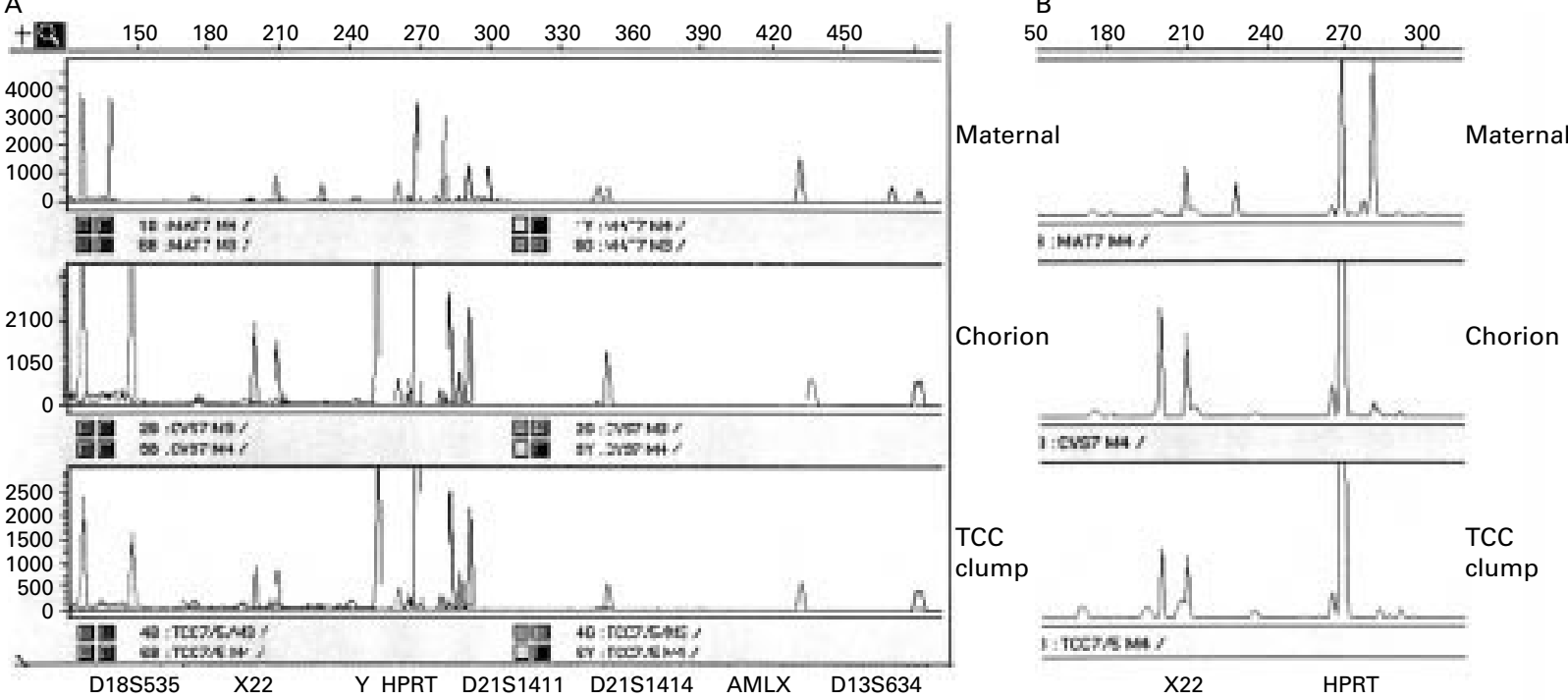

Figure 1 (A) Electrophoretogram of maternal, CVS, and TCC clump tested with X22, AMXY, and HPRT markers and other autosomal STRs (see text). (B) Electrophoretogram of maternal, CVS, and TCC clump tested with X22 and HPRT markers.

from these clumps can be used for the prenatal diagnosis of inherited single gene disorders. ${ }^{25}{ }^{5}$ The X22 pentanucleotide repeat is also of diagnostic value for the detection of cells present in maternal blood derived from female fetuses.

M ADINOLFI
V CIRIGLIANO

Galton Laboratory, University College London, 4 Stephenson Way, London NW1 3HE, UK

Correspondence to:

Professor Adinolfi, matteo@galton.ucl.ac.uk
1 Adinolfi M. Non- or minimally invasive prenatal diagnostic tests on maternal blood samples or transcervical cells. Prenat Diagn 1995;15:889-97.

2 Adinolfi M, Sherlock J. First trimester prenatal diagnosis using transcervical cells: an evaluation. Hum Reprod 1997;3:383-92.

3 Kingdom J, Sherlock S, Rodeck C, Adinolfi M. Detection of trophoblastic cells in transcervical samples collected by lavage or cytobrush. Obstet Gynecol 1995;86:283-8

4 Cirigliano V, Sherlock J, Conway G, Quilter C, Rodeck C, Adinolfi M. Rapid detection of chromosome $\mathrm{X}$ and $\mathrm{Y}$ aneuploidies by quantitative fluorescent PCR. Prenat Diagn 1999;19:1099-103.

5 Sherlock J, Cirigliano V, Petrou M, Tuuschek B, Adinolfi M. Assessment of diagnostic fluorescent multiplex polymerase chain reaction assay performed on single cells. Ann Hum Genet 1998;62:9-23.

6 Adinolfi M, El-Hashamite N, Sherlock J, Ward RHT, Petrou M, Rodeck C. Prenatal detection of haemoglobinopathies using transcervical cells. Prenat Diagn 1997;17:539-43. 